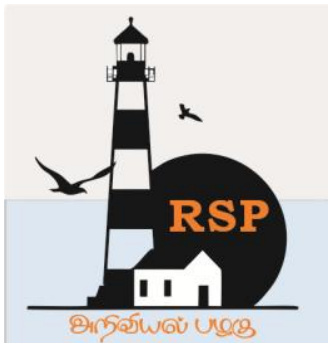

INTERNATIONAL RESEARCH JOURNAL ON ADVANCED SCIENCE HUB

\title{
Light Communication Applications Based on LIFI
}

B.Farhan ${ }^{1}$, S.Aasha ${ }^{2}$

${ }^{1}$ Research Scholar, Department of English Literature, AIMAN College of Arts and Science for Women, Trichy, India

${ }^{2}$ Assistant Professor, Department of Computer Science, AIMAN College of Arts and Science for Women, Trichy, India farhaneducate@gmail.com ${ }^{1}$

\section{Abstract}

Li-Fi which is also known as Light Fidelity refers to $5 G$ light Communication systems which uses lightemitting diodes bulb as a medium for high-speed data communication instead of WI-FI. Nowadays internet has become a serious demand, people are using Wi-Fi hotspot in all public places.In the epoch of internet, there is a continuous urge for faster, secure, and reliable wire-wireless connectivity in all fields, while wireless networks are more preferable in all domestic application in general. Li-Fi is a new digital communication may be a best and alternative to Wi-Fi in wireless communication. This paper proposes about what is Li-Fi Technology, working of Li-Fi, advantages and its applications. The Li-Fi technology was proposed by Professor Harald Hass in University of Edinburgh. Li-Fi has thousand-fold greater speed than Wi-Fi and provides greater security because the light is unable to penetrate through the walls, which propose a replacement era of wireless communication. The concept of Li-Fi is data communication is acheived on fast flickering of LED light, which is not detected by human eye but it can be received on photo diode receiver which converts the on-off state into binary digital data. It has gained an enormous popularity in two years of its invention. Such technology has brought not only greener but safer and cheaper way forward for communication.

Keywords: LED (Light Emitting Diode), Wi-Fi (Wireless Fidelity), Li-Fi (Light Fidelity), VLC (Visible Light Communication), RF (Radio Frequency)

\section{Introduction}

In the era of overcrowded data communication world, Li-Fi may be a new way of wireless communication that uses LED lights to transmit data wirelessly. Transmission of data is one among the foremost important day to day activities within the fast growing world. The current wireless networks that connect us to the web are very slow when multiple devices are connected.
Also with the rise within the number of devices which access the web, the supply of fixed bandwidth makes it far more difficult to enjoy high data transfer rates and to prevent from attacks and provide a secure network. Radio waves are just a little a part of the spectrum available for data transfer. Li-Fi possesses a way broader spectrum for transmission compared to standard methods of wireless communications that believe radio waves. 


\section{www.rspsciencehub.com}

The basic ideology behind this technology is that the info are often transferred through LED light by varying light intensities faster than the human eyes can perceive. This technology uses a neighbourhood of the spectrum that's still not greatly utilized. The concept of $\mathrm{Li}-\mathrm{Fi}$ was proposed by a German physicist Harald Hass within the TED (Technology, Entertainment, and Design) Global talk on light Communication (VLC) in July 2011, by pertaining to it as "data through illumination". He used a lamp with an LED bulb to transmit a video of a blooming flower that was then projected onto a screen. In Simple terms, In place of Wi-Fi modems, Li-Fi would use transceivers fitted with LED lamps that would light an area also as transmit and receive information. By adding new and unutilized bandwidth of light to the currently available radio waves for data transfer, Li-Fi can play a serious role in relieving the heavy loads which the current wireless system is facing.

By Communication through light, Li-Fi technology has the likelihood to vary how we access the web, stream videos, receive emails and far more. Security would not be an issue as data cannot be accessed in the absence of light. As a result, it is often utilized in high security military areas where RF communication is susceptible to eavesdropping. The opportunity to take advantage of a totally different a part of the spectrum is extremely appealing. Li-Fi has other advantages over Wi-Fi, like safe to use at atomic power plants, thermal power stations where Wi-Fi cannot be used. In such stations RF waves can be harmful and may cause accident, to speak in such regions only light spectrum are often safe. Apart from adverse regions $\mathrm{Li}-\mathrm{Fi}$ also can be utilized in all places where even $\mathrm{Wi}-\mathrm{Fi}$ is cannot be used.

\section{Literature Survey}

Dr. Harald Hass provided a deep insight in this technology, He introduced an illustration of $\mathrm{Li}-\mathrm{Fi}$ in the year 2011 at TED Global Conference in Edinburgh, and he demonstrated the use of Li-Fi
Volume 02 Issue 07 July 2020

and advantages of Li-Fi over Wi-Fi. His research led many people to work upon this technology [1].

After that Liang Yin (student member IEEE) together with Prof. Harald Hass made an attempt to show the clear difference between visible light communication (VLC) and light-fidelity (LiFi),Further they enlightened us how LiFi takes VLC by the use of light emitting diodes [2].

Harald Hass in the month of December in year 2013 worked upon the Li-Fi modulation and networked Li-Fi attocell concept, along with Prof. Svilen Dimitrov, Prof.ThiloFath, Prof. Irina Stefan and many others contributed to make this technology a big success [3].

EugeneCNelson professor, Elena E ftimovska researcher in the year 2014 researched about the Clinicians understanding of the effect of disease and treatment on patient's daily lives is poor. In response to this problem, over the past three decades, hundreds of standard is measures have been developed to capture patient reported outcomes, including symptom status, physical function, mental health, social function, and well being. However, the patient reported outcome measures (PROMs) movement has largely been driven by the agenda of researchers or service payer sandhas failed to focus effectively on improving the quality of care from the patient's perspective [4].

\section{Working Principle of Li-Fi}

Li-Fi uses light as a medium for the transmission of knowledge. As a kind of VLC system, it requires two components: a photodiode and a light-weight source. The photodiode acts as a transceiver that receives light signals and transmits them back. The LED light source transmits data using emitted light to the medium. During this case, light emitting diodes (LED) will function as LED light source. They are outfitted with a chip that is the signal processing unit. 


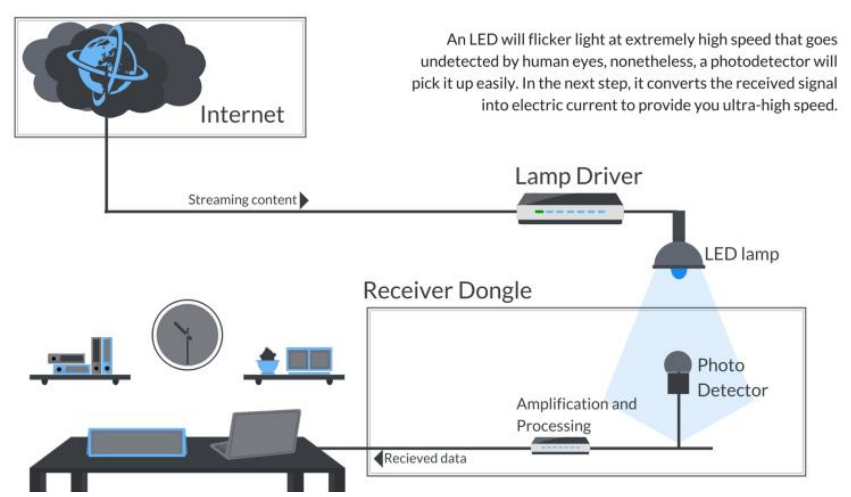

Fig.1. Working of LI-FI

LED light bulbs are semiconductors. This suggests current supplied to the bulb are often modulated, which successively, modulates the LED light they emit. This process occurs at extremely high speeds that are unperceivable to the human eye. Data is fed into the LED light bulb and sends the data's at extremely high speeds to the photodiode. It converts the data received into a binary data and it stream perceivable by humans like video and audio applications.

\section{How Data Is Transmitted Using LI-FI}

To send data over light, Li-Fi systems require a robust, robust light like LED bulbs. LEDs are different from halogen or filament bulbs as they are doing not got to warm up. As previously stated, they are semiconductors. They begin up quickly and emit light consistent with the present skilled them.

Within the LED light, the intensity of the colours red, green, and blue (RGB) is finely modulated to embed data into the LED light. (Again, this process is undetectable to the eye). This fine modulation of RGB is often better described as a sort of code. Once the LED light is received by a photodiode, the LED light is demodulated. The knowledge received is either relayed to a cloud server or transcribed by the receiver itself. Content is then displayed consistent with the code obtained.

\section{APPLICATIONS BASED ON LIFI}

\subsection{Blind Indoor Navigation System}

Indoor navigation is one such blessing that can be convenient for everybody, and it's especially indispensable for the visually impaired. We proposed such a navigation system for the visually impaired as shown in Figure.5.1 LED lights emit light with location data and embedded system or Smartphone with a clear light receiver receives the info. The embedded system calculates the optimal path to a designation and speaks to the visually impaired through a headphone.

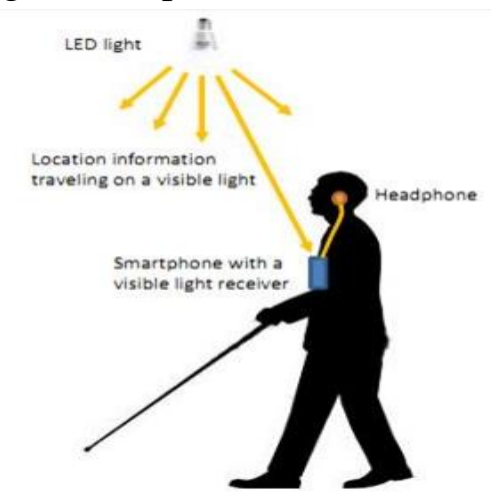

Fig.2. Blind Indoor Navigation System

\subsection{Under Water Communication System}

Information is transmitted from one place to another via modulation. Modulation is the process of communication, is that the process of transmission of low frequency data signal with high frequency carrier signal. Data such as voice, music, map, and video is transmitted and high frequency carrier signal. For 3 reasons modulation may be a necessity. First, low frequency data signal has not that much energy to travel in far distances. Second, if low frequency data signal were not imposed on carrier signal, in other words if not modulated, the dimension of the antenna would be inefficiently long. It's because the dimension of the antenna is inversely proportional to frequency. Third, data signal bandwidth is $20 \mathrm{~Hz}-20 \mathrm{KHz}$ and assuming the frequency range of $\mathrm{AM}$ is $5-10 \mathrm{KHz}$, there might be a couple of stations established. For these causes modulation as basis of communication may be a demanding tool needed to be used. 
www.rspsciencehub.com

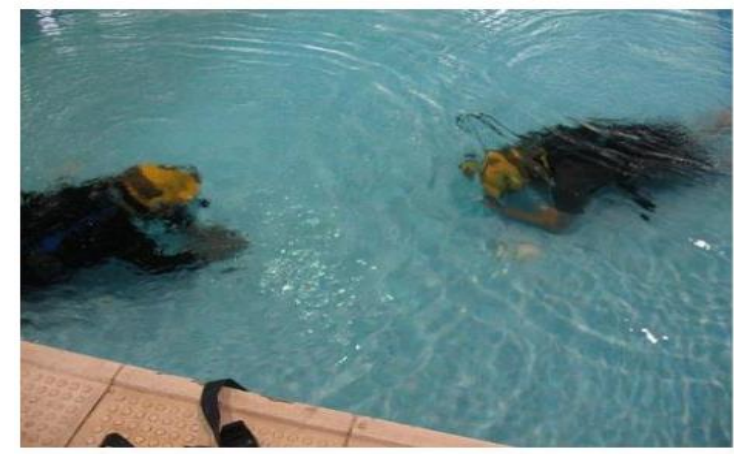

Fig.3. under Water Communication System

\subsection{Preventing Phishing Attacks using One Time}

\section{Password and User Machine Identification}

Phishing may be a sort of attack during which cyber criminals tricks the victims to steal their personal and financial data. It has become an organized criminal activity. To rescue the victims from revealing their personal data's, financial data by misdirecting them to the counterfeit website. Spoofed emails claiming to be from legitimate source are crafted. This research paper presents a completely unique approach to combat the Phishing attacks. An approach is proposed where user will retrieve the just one occasion password by LiFi Device LCD screen. After receiving the one time password the application software will create an encrypted token for the user's computer/device for authentication. The encrypted token are going to be used for identification, any time user wishes to access the web site he/she must request the new password. The just one occasion password as name implies will expire after single use. The just one occasion password and encrypted token may be a smart thanks to tackle this problem.

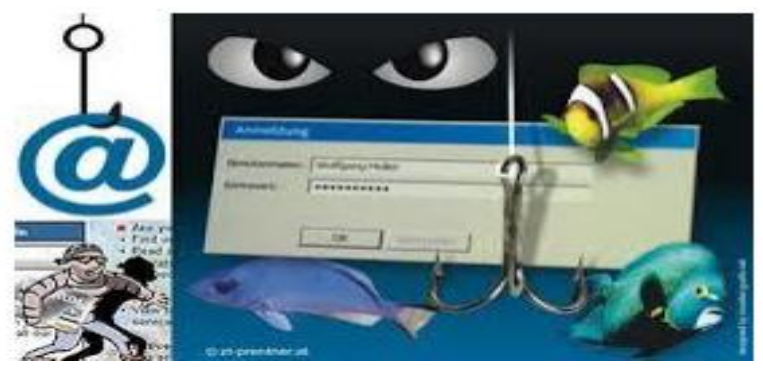

Fig.4 Preventing Phishing Attacks
Volume 02 Issue 07 July 2020

5.4 Super Market Navigation System and Discount

\section{Information Based On Location}

Positioning, also referred to as localization, is that the process of determining the spatial position of an object or person. Accurate positioning is critical for various applications. The familiar Global Positioning System originally a U.S. military system, is now in everyday use round the world, often in new and unexpected ways. Unfortunately, GPS isn't suitable in many indoor situations. To acquire location information using GPS, a tool must be ready to receive signals from different types of GPS satellites; It is not going to be accurate enough for several indoor applications. Despite decades of research into indoor positioning using technologies like radio systems supported wireless local area networks (LANs), there's still no system that's cheap, accurate, and widely available. The elemental problem in radio based systems is multipath propagation. Radio wave signals may reach a receiver by both direct line of sight and multiple reflected paths. This suggests that there is no simple and reliable way of determining the space or direction of the transmitter from the received signal.

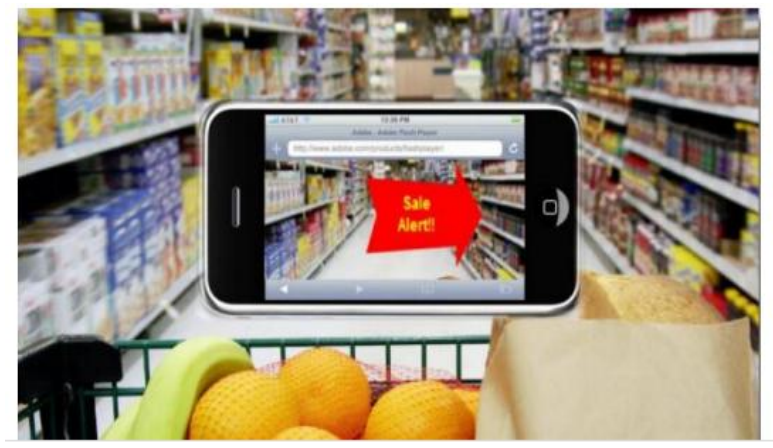

Fig.5 Super Market Navigation System

The introduction of white LEDs widespread for illumination provides an unprecedented opportunity for visible light positioning (VLP) to fill this gap, and form the idea for a widely available, economical, and easy-to-use indoor system. Look up in almost any building and you'll be ready to see multiple light fittings, demon starting that at the most indoor location, a receiver 


\section{www.rspsciencehub.com}

might be designed to receive line-of-sight signals from multiple light sources. The introduction o LED lighting creates a replacement opportunity for creating an inside positioning system. This was no possible with conventional lighting, but LEDs have a number of key advantages. First, LEDs are often modulated at much higher frequencies than conventional lighting; therefore the signals required for positioning can readily be transmitted at frequencies that don't cause visible flicker. Second, although LED lights are initially costlier, they need a way longer life-time, typically several years. This means that the added cost of constructing lights with the extra functionality required for positioning will be relatively smaller, and the benefits longer lasting.

\subsection{Smart LIFI Based Car Parking System}

Smart parking system exploits visible light communication (VLC) technology to assist drivers getting the real-time parking information also as direction guide. By providing correct information on available parking spaces, drivers save time and fuel and increase efficiency of the parking process. The proposed system not only produces the illumination function of LED but also the function of communicating within the way of application supported the VLC. The effectiveness of the proposed scheme is validated through experiments in an interior environment.

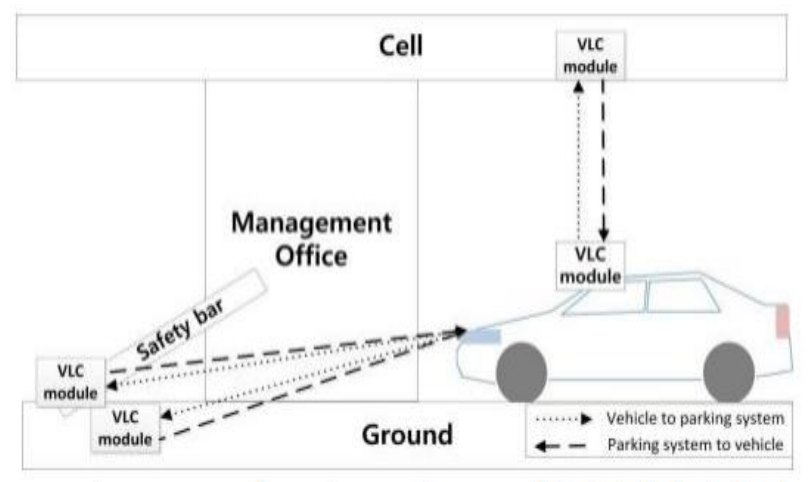

Fig.6. Smart LIFI Based Car Parking System

In modern life, the shopping complexes always attract people for its good products and repair.
Volume 02 Issue 07 July 2020

What's more, shopping complexes have begun providing services much more diverse than

\subsection{Positioning Techniques For Accurate Localization}

Mobile robot navigation when accurate positioning is required, as within the mobile robot example of Fig. 3, the VLP receiver will use the received signals to see the relative distance and/or direction of variety of LED transmitters. The measurements found will be be combined using classical triangulation (using angle of arrival information) or trial alteration (using path length or time of arrival information) to see the position of the receiver.

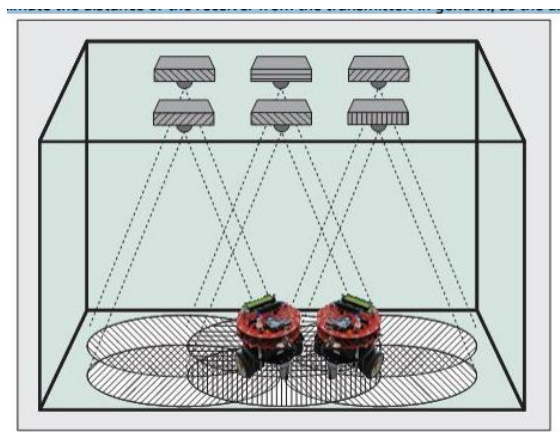

Fig.7 Positioning Techniques

We now discuss a variety of positioning techniques and their suitability to be used with lighting LEDs. Many of the indoor positioning systems that are supported radio signals use received signal strength (RSS) to estimate the space of the receiver from the transmitter. In general, because the distance between transmitter and receiver increases, the facility of the received signal falls. However, the consequences of objects blocking and reflecting the radio wave mean that the connection between distance and RSS is unpredictable, limiting the accuracy of an RSS approach in radio-based systems.

\section{ADVANTAGES OF LI-FI:}

$\mathrm{Li}-\mathrm{Fi}$, which uses light to transmit signals wirelessly, is an emerging technology poised to compete with Wi-Fi. Also, Li-Fi removes the restrictions that are placed on 
www.rspsciencehub.com

the user by the radio emission transmission like Wi-Fi as explained

a) Efficiency: Energy consumption are often minimized with the utilization of LED illumination which are already available within the home, offices and Mall etc. for lighting purpose. Hence the transmission of knowledge requiring negligible additional power, which makes it very efficient in terms of costs also as energy.

b) High speed: High Combination of low interference, high bandwidths and highintensity output, help Li-Fi provide high data rates i.e. $1 \mathrm{Gbps}$ or maybe beyond.

c) Availability: Availability is not a problem as light sources are present everywhere. Wherever there's a light-weight source, there is often Internet. Light bulbs are present everywhere - in homes, offices, shops, malls and even planes, which may be used as a medium for the data transmission.

d) Cheaper: Li-Fi not only requires fewer components for its working, but also uses only a negligible additional power for the info transmission.

e) Security: Li-Fi is major advantage to be considered in security. Since light cannot undergo opaque structures, $\mathrm{Li}-\mathrm{Fi}$ internet is out there only to the users within a confined area and can't be intercepted and misused, outside the world under operation.

\section{Future Scope:}

Li-Fi technology features a great scope in future. The extensive growth within the use of LEDs for illumination indeed provides the chance to integrate the technology into a environments and applications.

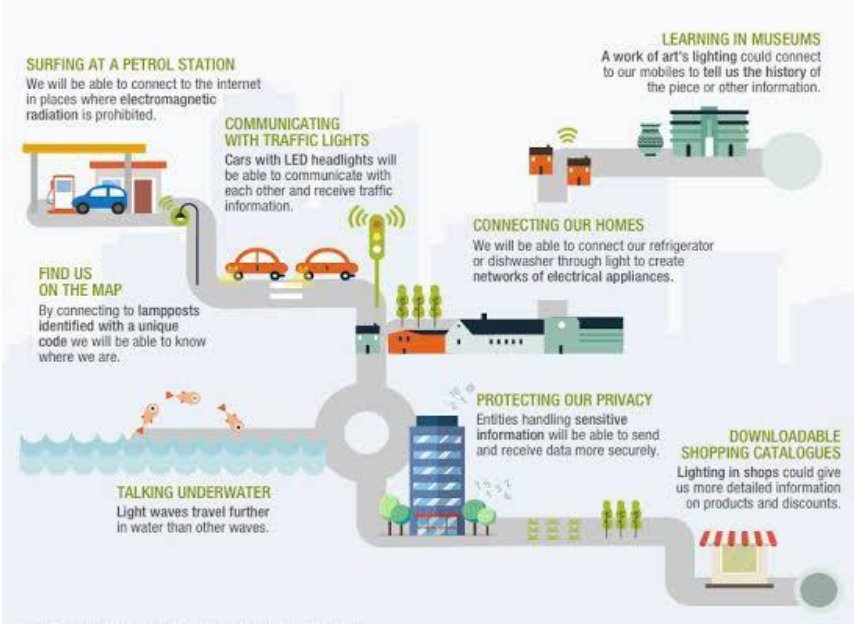

As light is everywhere and liberal to use, there's an excellent scope for the utilization and evolution of LiFi technology. If this technology becomes mature, each Li-Fi bulb is often wont to transmit wireless data. Because the Li-Fi technology becomes popular, it'll cause a cleaner, greener, safer communications and have a bright future and environment. The concept of $\mathrm{Li}-\mathrm{Fi}$ is deriving many of us because it is free (require no license) and faster means of knowledge transfer. If it evolves faster, people will use this technology more and more. Li-Fi Currently, LBS (location Based Service) or Broadcast solution are commercially available. Subsequent step might be a Li-Fi WLAN for B2B market with high added value on specific business cases and will grow towards mass market. Within the future, The LiFi could become an alternate solution to radio for wireless high rate room connectivity and new adapted service, like augmented or computer game.

\section{Conclusion}

As light is everywhere and liberal to use, there's an excellent scope for the utilization and evolution of $\mathrm{Li}-\mathrm{Fi}$ technology. If this technology becomes mature, each Li-If bulb is owns to transmit wireless data. It will cause a cleaner, greener, safer communications and have a bright future and 
www.rspsciencehub.com

environment. The concept of Li-Fi is driving many of us since it is free (require no license) and faster, provides means of data transfer. Hence People will use this technology more and more. $\mathrm{Li}-\mathrm{Fi}$ is currently implemented in LBS (location Based Service) or Broadcast solution is commercially available. The $\mathrm{Li}-\mathrm{Fi}$ could become an alternate solution to radio for wireless high rate room connectivity and new adapted service, like augmented or computer game.

\section{Reference}

[1]. Dr. Harald Haas, "wireless data from every light bulb”, TED Global, Edinburgh, July 2011

[2]. Harald Haas, Liang Yin,: 'What is Li-Fi?' published in journal of light wave technology, Volume: 34, Issue:6, March 15, 2016.

[3]. Harald Haas with Prof Svilen and Prof Thilo and Prof Irina: "Li-Fi modulation and networked Li-Fi attocell concept" 2012.

[4]. Kalpana.P.M, "Design and Development of PIC Microcontroller based Wireless Architecture for Human Health Monitoring", International Journal of Innovative Research in Science, Engineering and Technology Vol. 4, Issue 4, April 2015

[5]. "Visible-light communication" International Journal of Engineering Trends and Technology ISSN: 2231-5381 Volume-4 Issue- 3, 2013

[6]. Light-Fidelity: A Reconnaissance of Future Technology International Journal of Advanced Research in Computer Science and Software Engineering ISSN: 2277 128X Volume 3, Issue 11, November 2013

[7]. Next of Wi-Fi an Future Technology in Wireless Networking Li-Fi Using Led Over Internet of Things International Journal of Emerging Research in Management
Volume 02 Issue 07 July 2020

\&Technology ISSN: 2278-9359 (Volume-3, Issue-3) March 2014

[8]. "Li-Fi Technology" Transmission of data through light Int.J.Computer Technology \& Applications, ISSN: 2229-6093 Vol 5 (1),150154 jan - feb 2014

[9]. "Embedded Patient Monitoring System" International Journal of Embedded Systems and Applications (IJESA) Vol.1, No.2, December 2011

[10]. "Embedded Based Real-time Patient Monitoring System International Journal of VLSI and Embedded Systems-IJVES ISSN: 2249 - 6556 Vol 05, Article 02231; March 2014 